ОБЕСПЕЧЕНИЕ ЭЛЕКТРОМАГНИТНОЙ БЕЗОПАСНОСТИ НА ВЫСОКОСКОРОСТНОМ МАГЛЕВ ТРАНСПОРТЕ

\author{
С. М. Аполлонский \\ ООО «Центр электромеханотроники» \\ (Санкт-Петербург, Россия)
}

\title{
ENSURING ELECTROMAGNETIC SAFETY AT HIGH-SPEED MAGLEV TRANSPORT
}

\author{
S. M. Apollonskiy \\ Ltd. "Center of Elektromehanotroniki" \\ (St. Petersburg, Russia)
}

В докладе рассмотрены подходы к снижению загрязнения техносферы электромагнитными полями (ЭМП) от высокоскоростного МАГЛЕВ транспорта. Рекомендована математическая модель компоновки элементов электроэнергетической установки на МАГЛЕВ транспорте с целью минимизации загрязнения окружающей среды от ЭМП.

Высокоскоростной наземный транспорт (ВСНT) в современном понимании - это железнодорожный транспорт, обеспечивающий движение со скоростью более 200 км/ч. ВСНТ осуществляется либо колесным подвижным составом по рельсовому пути, либо бесконтактным способом, когда для тяги и торможения применяется линейный электрический привод, а для создания условий движения - магнитный подвес, так называемый левитирующий транспорт. Таковым является ВСНТ МАГЛЕВ.

При движении ВСНТ МАГЛЕВ создаёт помехонесущие ЭМП широкого частотного диапазона, распространяющиеся как в окружаюшей внешней среде, так и внутри самого движушегося средства [1].

В математическом аспекте ЭМП представляют собой векторные (а в отдельных случаях и тензорные поля), где 
функциями являются магнитные и электрические напряженности. Возникает задача по обеспечению электромагнитной безопасности (ЭМБ) ВСНТ МАГЛЕВ.

Технический регламент о безопасности высокоскоростного железнодорожного транспорта [2] устанавливает минимально необходимые требования к ВСНТ, выполнение которых обеспечивает ЭМБ, виброакустическую безопасность и др. Эти требования относятся и к ВСНТ МАГЛЕВ, ЭМП которого, рассеиваемые в окружающей среде, могут быть соизмеримыми с ЭМП рельсового ВСНТ.

При обеспечении проблемы ЭМБ каждое из устройств электроэнергетической установки (ЭЭУ) ВСНТ МАГЛЕВ следует рассматривать как элемент некоторой подсистемы, в которой проявляются негативные связи (электрические и магнитные) этого элемента с другими. Такой подход позволяет рассматривать проблему ЭМБ как общую проблему при исследовании источников и рецепторов ЭМП, выделяя в них соответствующие признаки, которым ранее не придавалось значения. ЭМБ представляет собой область науки и техники, развитие которой тесно связано с электротехникой и электроникой.

Для снижения негативного воздействия ЭМП на техносферу как внутри ВСНТ МАГЛЕВ, так и вне его, необходимо соответствующим образом синтезировать его ЭЭУ.

В процессе синтеза элементов ЭЭУ, характеристики которых зависят от поведения ЭМП, возникают задачи оптимизации размещения источников ЭМП в областях с изменяемыми геометрическими характеристиками. В связи с этим важно, чтобы математическая модель проектируемого устройства, кроме технологических и конструктивных ограничений, одновременно учитывала и отмеченные выше факторы.

С целью снижения загрязнения окружающей среды ЭМП при проектировании ЭЭУ ВСНТ МАГЛЕВ до нормативных значений необходимо поставить задачу о рациональном размещении источников ЭМП ЭЭУ. Такой подход позволит сформировать структуру помехонесущего ЭМП от ЭЭУ в нормативных пределах. Особое значение такой подход приобретает в связи с тем, что ЭЭУ ВСНТ МАГЛЕВ находится в 
металлическом корпусе, являющимся экранирующей структурой, но недостаточной для существенного снижения помехонесущих ЭМП, выходящих во внешнее пространство.

Применение для поддержания ЭМП в заданных пределах дополнительных активных и пассивных экранов не всегда возможно из-за больших габаритов и весов последних и из-за конструктивно-технологических трудностей их создания и размещения. Поэтому на первое место выступает задача рационального размещения элементов ЭЭУ внутри энергетического помещения. Такой подход поможет решить проблему снижения помехонесущих ЭМП от ЭЭУ ВСНТ МАГЛЕВ до требований ЭМБ.

Для решения подобной задачи необходимо иметь, с одной стороны, метод расчета ЭМП от нескольких источников, расположенных в пределах энергетического помещения, a c другой - методы формализации и алгоритмы решения оптимизационных задач размещения [3].

Выяснение вопросов оптимального размещения источников ЭМП, имеющих определенные геометрические и энергетические характеристики, имеет значение для практики проектирования ЭЭУ. При этом поиск наилучшего размещения источников ЭМП должен осуществляться с учетом ограничений как на характер ЭМП, так и на местоположение источников поля.

Разработке математических моделей и методов решения задач о размещении источников физических полей посвящена работа [4]. Однако, в ней не затрагивались вопросы размещения источников ЭМП. Отдельные вопросы, связанные с оптимизацией размещения источников ЭМП, рассматривались лишь в работах по проектированию радиоэлектронной аппаратуры [5].

Отметим, что математическая постановка большинства задач оптимального размещения источников ЭМП приводит к нелинейным задачам математического программирования специального вида. Особенности такого класса задач не позволяют воспользоваться известными методами математического программирования. Кроме того, поиск оптимального размещения источников необходимо осуществлять с учетом их взаимовлияния, т.е. рассматриваемый класс задач относится к задачам оптимизации систем 
многосвязного управления [6]. При этом взаимовлияние обусловлено физическими свойствами управляемого объекта, и применение принципов автономности [7] затруднено.

Следует помнить, что разработка методов и алгоритмов решения задач оптимального размещения источников физических полей, а также создание соответствующего математического обеспечения (комплекса программ) имеет значение для всех областей, в том числе и электродинамики. Это связано с тем обстоятельством, что во многих областях инженерной практики возникают задачи оптимального размещения объектов, являющихся источниками соответствующих физических полей или влияющих на характер протекания физических процессов. Тем не менее, задачи оптимального размещения источников ЭМП мало затронуты в публикациях [8-9].

ЭМП элементов ЭЭУ, создаваемые большим числом источников различной напряженности и геометрической формы, являются весьма сложными по своей структуре. При этом обеспечение ЭМБ группы элементов имеет значение, поэтому поиск конструктивной топологии элементов часто приходится осуществлять на множестве тех вариантов, которые удовлетворяют условиям ЭМБ ЭЭУ.

Ниже рассмотрены задачи оптимизации ЭМП группы источников в экранирующих структурах с учетом их рационального размещения. Решение таких задач стало возможным, благодаря ряду предложений, внесенных автором доклада: введения скалярных потенциалов для описания внешних ЭМП элементов ЭЭУ; введения функций взаимовлияния, учитывающих влияние соседних источников; разработке экранирующих функций в виде разложений по гармоникам; использования теорем сложения, позволяющих решения уравнений математической физики представлять в разных системах координат [3]. Библиографический список насчитывает 14 источников. 


\section{Сведения об авторе:}

Аполлонский Станислав Михайлович, доктор технических наук, профессор, заслуженный деятель науки РФ, научный консультант ООО «Центр электромеханотроники», контакты: 197110, Санкт-Петербург, Малый пр. ПС, дом 36-38, кв. 16, тел. 235-55-31, E-mail: smapollon@yahoo.com.

\section{Information about author:}

Stanislav M. Apollonskiy, Doctor of Technical Sciences, Professor, Honored Worker of Science, Research consultant Ldt. "Center of Elektromehanotroniki". Contacts: 197110, St. Petersburg, Maly prospect P.S., H. 36-38, Ap. 16, tel. 235-55-31, E-mail:smapollon@yahoo.com. 